\title{
NON-EQUILIBRIUM PHASE TRANSITIONS, COHERENCE AND CHAOS
}

\author{
GEOFFREY L. SEWELL \\ Department of Physics \\ Queen Mary and Westfield College \\ Mile End Road, London E1 4NS, England \\ E-mail: G.L.Sewell@qmw.ac.uk
}

\begin{abstract}
We present a scheme for the theory of phase transitions in open dissipative systems, and show that its demands are fulfilled by quantum stochastic models of open systems, such as the laser.

1. Introduction. Phase transitions far from equilibrium abound in physics [1-3], chemistry [3] and biology [4], and are indeed crucial to fundamental questions concerning the generation of ordered and chaotic structures in the natural sciences. The quantum theory of these transitions, however, is still rather undeveloped at the level of mathematical physics, being confined to a few special models, such as those of a laser [5-7], a plasma [8] and a cell [9].

The object of this article is to propose a scheme for a general, if rudimentary, framework for such a quantum theory, and to provide a concrete example where it is fully realised.

We shall start, in Section 2, by formulating our schemes for the theories of phase structures both of equilibrium states of conservative systems and non-equilibrium states of open dissipative ones, within the terms of the algebraic framework of quantum statistical mechanics. In Sections 3 and 4, we shall provide a resume of how our scheme for nonequilibrium phase structures is fulfilled by a recent and new version of the Dicke- HeppLieb laser model, that was formulated [6] in terms of quantum dynamical semigroups and stochastic processes. Specifically, we shall present the main features of the model in Section 3, and in Section 4 we shall describe our results $[6,7]$ on its transitions between normal, coherent and chaotic phases. We shall conclude, in Section 5, with a brief discussion of our main conclusions and of how they may be extended to other models.
\end{abstract}

1991 Mathematics Subject Classification: Primary 82C26; Secondary 46L55.

The paper is in final form and no version of it will be published elsewhere. 


\section{General consideration of phase structures}

2.1. The generic model. We take this to be a quantum dynamical system $\Sigma=$ $(\mathcal{A}, \mathcal{S}, T)$, where

(a) $\mathcal{A}$ is a *-algebra of observables;

(b) $T$ is a representation of either $\mathbf{R}$ in $A u t(\mathcal{A})$ or of $\mathbf{R}_{+}$in the completely positive contractions of that algebra, according to whether the system is conservative or dissipative; and

(c) $\mathcal{S}$ is a space of states on $\mathcal{A}$, that is stable under the action of the dual of the $T$.

The physical interpretation of the triple $(\mathcal{A}, \mathcal{S}, T)$ is encapsulated by the specifications that the expectation value of an observable $A(\in \mathcal{A})$ in the state $\omega(\in \mathcal{S})$ is $\omega(A)$ and that the evolute of that observable at time $t$ is $A_{t}=T(t) A$. In general, $\Sigma$ corresponds to an infinitely extended system of particles of one or more species, and the construction of $(\mathcal{A}, \mathcal{S}, T)$ may be effected by standard procedures [10-12].

We shall now outline the description of both equilibrium and non- equilibrium phases of this generic model.

2.2. Equilibrium phases of conservative systems. This subsection consists of a sketch of our scheme, presented in Chapter 4 of [12], for the description of these phases.

We assume that there is a set, $\hat{x}=\left(\hat{x}_{1}, \ldots, \hat{x}_{m}\right)$, of linearly independent macroscopic observables of $\Sigma$, that are the global spatial averages of densities of locally conserved quantities (energy, particle number, momentum, etc.), ${ }^{*}$ which satisfies the following completeness condition. Given the expectation value, $x$, of $\hat{x}$, there is precisely one state, $\omega_{x}$, of the system that maximises its global entropy density.** We denote by $X$ the subset of $\mathbf{R}^{m}$ comprising the range of the expectation values of $\hat{x}$ : in fact, $X$ is convex.

Thus, $\omega_{x}$ is the thermal equilibrium state for which the expectation value of $\hat{x}$ is $x$. We define $s(x)$ to be the entropy density of state, and $f$ to be its Legendre transform: this is the function of the thermodynamic conjugates, $\theta=\left(\theta_{1}, \ldots, \theta_{m}\right)\left(\in \mathbf{R}^{m}\right)$, of the $x_{j}$ 's given by the standard formula

$$
f(\theta)=\sup _{x}(s(x)-x \cdot \theta) .
$$

In fact, the supremum here is actually attained, and the value of $\theta$ at a maximal point, $x$, is

$$
\theta=\frac{\partial s(x)}{\partial x}
$$

We denote by $\Theta$ the subset of $\mathbf{R}^{m}$, given by the range of the function $\partial s / \partial x$, i.e., of the variable $\theta$. Thus, $\Theta$ may be regarded as the macroscopic 'control space'. Note that the thermodynamic variables given by the components of $\theta(\in \Theta)$ are the inverse temperature, chemical potential, etc.

The phase structure of the system is related to the differentiability of $f$ in the following way (cf. Chapter 4 of [12]). A point $\theta$ of $\Theta$ corresponds to one or several points $x$ of

* These are 'observables at infinity', as defined by Lanford and Ruelle [13].

** The entropy density is defined as a functional on the state space [10, 12]. 
$X$, and correspondingly to one or several equilibrium states $\omega_{x}$, according to whether or not $f$ is continuously differentiable there. We assume, in accordance with classical thermodynamics [14] and results for tractable models, that the set of points where $f$ is not differentiable constitute surfaces that divide $\Theta$ into regions $\Theta_{1}, \Theta_{2}, \ldots$, etc. Thus, these regions correspond to the different phases of the system, and the phase transitions arise when their boundaries are crossed.

2.3. Non-equilibrium phases of dissipative systems. We shall now present a scheme for the description of these phases, that is realised by the laser model of Secs. 3 and 4, as well as other mean field models indicated in Section 5. This scheme is characterised by the following conditions.

(I) The dynamical semigroup, $T$, depends on a set of control variables, $\theta=\left(\theta_{1}, \ldots, \theta_{k}\right)$. However, by contrast with the conservative case, the $\theta_{j}$ 's are not generally of the thermodynamic type. For example, they could be parameters that govern the dissipative properties of the system, e.g. friction constants or strengths of energy pumps or sinks. We denote by $\Theta$ the space whose points are the accessible values of $\theta$.

(II) The system has a set of classical macroscopic observables $x=\left(x_{1}, \ldots, x_{m}\right)$, which is complete in the sense that the semigroup $T$ induces an autonomous dynamics of these variables, i.e., that $T(t) x$ is a function of only $x, t$ and the parameters of the model. Thus, defining $\tau(t)$ to be the restriction of $T(t)$ to $x$ and the functions thereof, and denoting by $X\left(\in \mathbf{R}^{m}\right)$ the range of the expectation values of $x,(X, \tau)$ is a classical dynamical system, $\mathcal{K}$. We assume that it has stable attractors (fixed points, periodic orbits, chaotic orbits), corresponding to domains $\Theta_{1}, \Theta_{2}, \ldots$ of the control space $\Theta$, and that it undergoes bifurcations corresponding to transitions between these when $\theta$ crosses the boundaries of these domains [15].

(III) The microscopic dynamics of $\Sigma$ is driven by the time-dependent macroscopic variable, $\tau(t) x=x_{t}$, and its microstate is determined by the instantaneous value of this variable when it lies an attractor of $\mathcal{K}$. We denote this state by $\omega_{x_{t}}$. We designate the phases of $\Sigma$ to correspond to the the domains $\Theta_{1}, \Theta_{2}, \ldots$ of $\Theta$, representing the attractors of $\mathcal{K}$. Thus, phase transitions occur when the control variable $\theta$ crosses the bifurcation sets given by the boundaries of these domains.

(IV) Although, as pointed out in (I), the variable $\theta$ is not generally thermodynamic, the form of the state $\omega_{x t}$ is governed by a maximum entropy principle. Specifically, this state maximises the entropy density of the system, subject to the contraints imposed by the instantaneous values of the macroscopic variables.

N ot e. Since, except for the stable fixed points, the attractors of the classical system $\mathcal{K}$ are not pointwise invariant under the dynamical semigroup $\tau$, the corresponding states, $\omega_{x_{t}}$, of $\Sigma$ are not stationary in time. By contrast, the thermal equilibrium states are, of course, stationary.

3. The laser model [6]. This model is a dissipative quantum system, $\Sigma$, consisting of a chain of identical atoms interacting with an $N$-mode radiation field. We build the model from its constituent parts as follows. 
3.1. The single atom. This is assumed to be a two-state atom or spin, $\Sigma_{a t}$. Its algebra of observables, $\mathcal{A}_{a t}$, is that of the two-by-two matrices, and is thus the linear span of Pauli matrices $\left(\sigma_{x}, \sigma_{y}, \sigma_{z}\right)$ and the identity, $I$. Its algebraic structure is provided by the relations

$$
\sigma_{x}^{2}=\sigma_{y}^{2}=\sigma_{z}^{2}=I ; \sigma_{x} \sigma_{y}=i \sigma_{z}, \text { etc. }
$$

We define the spin raising and lowering operators

$$
\sigma_{ \pm}=\frac{1}{2}\left(\sigma_{x} \pm i \sigma_{y}\right)
$$

We assume that the atom is coupled to a pump and a sink, and that accordingly [6] its dynamics is given by a one-parameter semigroup $\left\{T_{a t}(t) \mid t \in \mathbf{R}_{+}\right\}$of completely positive, identity preserving contractions of $\mathcal{A}_{a t}$, whose generator, $L_{a t}$, is of the following form.

$$
L_{a t} \sigma_{ \pm}=-\left(\gamma_{1} \mp i \epsilon\right) \sigma_{ \pm} ; L_{a t} \sigma_{z}=-\gamma_{2}\left(\sigma_{z}-\eta I\right),
$$

where $\epsilon(>0)$ is the energy difference between the ground and excited states of an atom, and the $\gamma$ 's and $\eta$ are constants whose values are determined by the atomic coupling to the energy source and sink, and are subject to the restrictions that

$$
0<\gamma_{2} \leq 2 \gamma_{1} ;-1 \leq \eta \leq 1
$$

In particular, $\eta$ is positive or negative according to whether the coupling of the atom to the pump or the sink is the stronger. In the former case, these couplings drive the atom to a terminal mixed state with inverted population, i.e. with higher occupation probability for the excited state than for the ground state.

3.2. The matter. This consists of a chain of copies of $\Sigma_{a t}$, located at the sites of the one-dimensional lattice $\mathbf{Z}$. We construct the model $\Sigma_{m a t}$ of the matter as a $C^{\star}$-dynamical system, $\left(\mathcal{A}_{\text {mat }}, \mathcal{S}_{\text {mat }}, T_{\text {mat }}\right)$, where $\mathcal{A}_{\text {mat }}$ is its algebra of quasi-local observables, $\mathcal{S}_{\text {mat }}$ is the space of states on $\mathcal{A}_{\text {mat }}$ and $T_{\text {mat }}$ is a one-parameter semigroup of CP transformations of this algebra, representing the dynamics of matter, when decoupled from the radiation.

To formulate $\mathcal{A}_{\text {mat }}$, we attach to each site, $r$, of $Z$ a copy, $\Sigma_{r}$, of $\Sigma_{a t}$, whose algebra of observables, $\mathcal{A}_{r}$, and dynamical semigroup, $T_{r}$, are isomorphic with $\mathcal{A}_{a t}$ and $T_{a t}$, respectively. We denote by $\sigma_{r, u}$ the copy of $\sigma_{u}$ at $r$, for $u=x, y, z, \pm$. We then $L$ to be the family of all finite point subsets of $\mathbf{Z}$, and we assign to each $\Lambda \in L$ the $C^{\star}$-algebra $\mathcal{A}_{\Lambda}:=\otimes_{r \in \Lambda} \mathcal{A}_{r}$. For $\Lambda \subset \Lambda^{\prime}$, we identify elements $A$ of $\mathcal{A}_{\Lambda}$ with $A \otimes I_{\Lambda^{\prime} \backslash \Lambda}\left(\in \mathcal{A}_{\Lambda^{\prime}}\right)$, thereby rendering $\mathcal{A}_{\Lambda}$ isotonic w.r.t. $\Lambda$. We then $\mathcal{A}_{\text {mat }}$ to be the completion of the local algebra $\mathcal{A}_{\text {mat }, L}:=\bigcup_{\Lambda \in L} \mathcal{A}_{\Lambda}$ w.r.t. the norm it inherits from the $\mathcal{A}_{\Lambda}$ 's. We denote by $\mathcal{A}_{\Lambda}^{\prime}$ the commutant of $\mathcal{A}(\Lambda)$ in $\mathcal{A}_{\text {mat }}$.

We define the elements $T_{\text {mat }}(t)$ of the dynamical semigroup $T_{\text {mat }}$ to be $\otimes_{r \in \mathbf{Z}} T_{r}(t)$. Thus, the generator, $L_{\text {mat }}$, of this semigroup is given by the formula

$$
L_{m a t}=\sum_{r \in \mathbf{Z}} L_{r}
$$

where

$$
\begin{gathered}
L_{r} \sigma_{r, \pm}=-\left(\gamma_{1} \mp i \epsilon\right) \sigma_{r, \pm} ; L_{r} \sigma_{r, z}=-\gamma_{2}\left(\sigma_{r, z}-\eta I\right) \\
\text { and } L_{r}\left(A_{r} A_{r}^{\prime}\right)=\left(L_{r} A_{r}\right) A_{r}^{\prime} \forall A_{r} \in \mathcal{A}_{r}, A_{r}^{\prime} \in \mathcal{A}_{r}^{\prime}
\end{gathered}
$$


We define $\Sigma_{\text {mat }}^{(N)}=\left(\mathcal{A}_{\text {mat }}^{(N)}, \mathcal{S}_{\text {mat }}^{(N)}, T_{\text {mat }}^{(N)}\right)$ to be the subsystem of $\Sigma_{\text {mat }}$ comprising the atoms in the segment $[-N, N]$ of $\mathbf{Z}$. Thus, $\mathcal{A}_{\text {mat }}^{(N)}=\mathcal{A}_{[-N, N]}$, and $\mathcal{S}_{\text {mat }}^{(N)}$ and $T_{\text {mat }}^{(N)}$ are the restrictions of $\mathcal{S}_{m a t}$ and $T_{m a t}$, respectively, to this algebra. The generator of $T_{m a t}^{(N)}$ is therefore

$$
L_{m a t}^{(N)}=\sum_{r=-N}^{N} L_{r}
$$

3.3. The radiation. We assume that the radiation field consists of $n(<\infty)$ modes, represented by creation and destruction operators $\left\{a_{l}^{\star}, a_{l} \mid l=0, \ldots, n-1\right\}$ in a Fock-Hilbert space $\mathcal{H}_{\text {rad }}$, as defined by the standard specifications that (a) these operators satisfy the CCR,

$$
\left[a_{l}, a_{m}^{\star}\right]=\delta_{l m} I ;\left[a_{l}, a_{m}\right]=0,
$$

and (b) $\mathcal{H}_{\text {rad }}$ contains a (vacuum) vector $\Phi$, that is annihilated by each of the $a$ 's and is cyclic w.r.t. the algebra of polynomials in the $a^{\star}$ 's.

We define the Weyl map $z=\left(z_{0}, \ldots, z_{n-1}\right) \rightarrow W(z)$, of $C^{n}$ into $\mathcal{L}\left(\mathcal{H}_{\text {rad }}\right)$ by the formula

$$
W(z) \equiv W\left(z_{0}, \ldots, z_{n-1}\right)=\exp \left[i\left(z . a+(z . a)^{\star}\right)\right], \text { with } z . a=\sum_{0}^{n-1} z_{l} a_{l} .
$$

We then define $\mathcal{A}_{\text {rad }}$ to be the ${ }^{\star}$-algebra of polynomials in the $a$ 's, $a^{\star}$ 's and the Weyl operators $W(z)$, with $z$ running through $\mathbf{C}^{n}$. Thus, in view of the CCR (3.9), this algebra is the linear span of the derivatives, of all orders, of the operators $W\left(t_{0} z_{0}, \ldots, t_{n-1} z_{n-1}\right)$ w.r.t. the real variables $\left(t_{0}, \ldots, t_{n-1}\right)$.

We assume that the radiation dynamics is given by the canonical extension to $\mathcal{A}_{\text {rad }}$ of Vanheuverszwijn semigroup [16], $T_{\text {rad }}$, of quasi-free CP transformations of the Weyl algebra of linear combinations of $\left\{W(z) \mid z \in \mathbf{C}^{n}\right\}$. The formal generator of this semigroup is

$$
L_{r a d}=\sum_{l=0}^{n-1}\left(i \omega_{l}\left[a_{l}^{\star} a_{l}, .\right]+2 \kappa_{l} a_{l}^{\star}(.) a_{l}-\kappa_{l}\left\{a_{l}^{\star} a_{l}, .\right\}\right),
$$

where $\{.,$.$\} denotes anticommutator, and the frequencies, \omega_{l}$, and the damping constants, $\kappa_{l}$, are positive.

3.4. The matter-radiation coupling. We pass now to the formulation of the model $\Sigma^{(N)}$, obtained by coupling $\Sigma_{m a t}^{(N)}$ to $\Sigma_{\text {rad }}$ via a certain dipolar interaction: we pass from this to the infinite system, $\Sigma$, in Section 4 .

We assume that the algebra of observables of $\Sigma^{(N)}$ is $\mathcal{A}^{(N)}:=\mathcal{A}_{\text {mat }}^{(N)} \otimes \mathcal{A}_{\text {rad }}$. Thus, $\mathcal{A}^{(N)}$, like $\mathcal{A}_{\text {rad }}$, is an algebra of both bounded and unbounded operators in the Hilbert space $\mathcal{H}^{(N)}:=\mathbf{C}^{4 N+2} \otimes \mathcal{H}_{\text {rad }}$. We identify elements $A, R$, of $\mathcal{A}^{(N)}$, $\mathcal{R}$, with $A \otimes I_{\text {rad }}$ and $I_{\text {mat }} \otimes R$, respectively.

We assume that the matter-radiation coupling is given by the interaction Hamiltonian

$$
H_{\text {int }}^{(N)}=i(2 N+1)^{-1 / 2} \sum_{r=-N}^{N} \sum_{l=0}^{n-1} \lambda_{l}\left(\sigma_{r,-} a_{l}^{\star} \exp (-2 \pi i l r / n)-h . c .\right),
$$

where the $\lambda$ 's are real-valued, $N$-independent coupling constants. Correspondingly, we define the radiation field, $\phi^{(N)}$, of the model by the stipulation that its value at the site 
$r$ is the coefficient of $\sigma_{r,+}$ in this formula. Thus,

$$
\phi_{r}^{(N)}=-i(2 N+1)^{-1 / 2} \sum_{l=0}^{n-1} \lambda_{l} a_{l} \exp (2 \pi i l r / n) .
$$

We now need to define the state space, $\mathcal{S}^{(N)}$, and the dynamical semigroup, $T^{(N)}$, in a way that takes account of the unboundedness both of $H_{\text {int }}^{(N)}$ and of some of the elements of $\mathcal{A}^{(N)}$. To this end, we start by defining $\mathcal{D}_{0}^{(N)}$ to be the space of density matrices in $\mathcal{H}^{(N)}$, with the trace norm topology, and $\mathcal{D}_{1}^{(N)}$ to be the subset of its elements, $\rho^{(N)}$, for which $\operatorname{Tr}\left(A^{\star} \rho^{(N)} A\right)$ is finite for all $A$ in $\mathcal{A}^{(N)}$. We define $\mathcal{S}_{1}^{(N)}$ to be the set of positive, normalised, linear functionals, $\psi^{(N)}$, on $\mathcal{A}^{(N)}$, that are in the one-to-one correspondence with the $\mathcal{D}_{1}^{(N)}$-class density matrices, given by the standard formula $\psi^{(N)}(A)=\operatorname{Tr}\left(\rho^{(N)} A\right)$. We then adopt the formulation of $T^{(N)}$ and $\mathcal{S}^{(N)}$ provided by [6], in which $T^{(N)}$ is constructed as the modification of $T_{m a t}^{(N)} \otimes T_{\text {rad }}$ due to the interaction $H_{\text {int }}^{(N)}$, and $\mathcal{S}^{(N)}$ is a 'large' subset of $\mathcal{S}_{1}^{(N)}$ that is stable w.r.t. this dynamical semigroup. Specifically, the construction yields the following results [6].

(1) The density matrices corresponding to $\mathcal{S}^{(N)}$ form a dense subset of $\mathcal{D}_{0}^{(N)}$, in the topology corresponding to the trace norm.

$(2) \mathcal{S}^{(N)}$ is stable under the transformations $\psi^{(N)} \rightarrow \psi^{(N)} \circ T^{(N)}(t):=\psi_{t}^{(N)}$.

$$
\frac{d}{d t} \psi_{t}^{(N)}(A)=\psi_{t}^{(N)}\left(L^{(N)} A\right) \forall A \in \mathcal{A}^{(N)}, t \in \mathbf{R}_{+},
$$

where

$$
L^{(N)}=L_{m a t}^{(N)}+L_{r a d}+i\left[H_{i n t}^{(N)}, .\right] .
$$

3.5. The macroscopic observables. We formulate the macroscopic description of the model, as in [6], in terms of the global intensive observables

$$
s_{l}^{(N)}=(2 N+1)^{-1} \sum_{r=-N}^{N} \sigma_{r,-} \exp (-2 \pi i l r / n) ; l=0, \ldots, n-1
$$

and

$$
p_{l}^{(N)}=(2 N+1)^{-1} \sum_{r=-N}^{N} \sigma_{r, z} \exp (-2 \pi i l r / n) ; l=0, \ldots, n-1,
$$

together with the operators

$$
\alpha_{l}^{(N)}=(2 N+1)^{-1 / 2} a_{l} ; l=0, \ldots, n-1,
$$

corresponding to a scaling of the number operators $a_{l}^{\star} a_{l}$ in units of $2 N+1$. Thus, by equns. (3.13) and (3.18), the radiation field may be re-expressed in the form

$$
\phi_{r}^{(N)}=-i \sum_{l=0}^{n-1} \lambda_{l} \alpha_{l}^{(N)} \exp (2 \pi i r l / n)
$$

We note that the set of $p^{(N)}$ 's is the same as that of their adjoints, since

$$
p_{0}^{(N) \star}=p_{0}^{(N)} ; \text { and } p_{l}^{(N) \star}=p_{n-l}^{(N)} \text { for } l=1, \ldots, n-1 .
$$


The set $M^{(N)}:=\left\{s_{l}^{(N)}, s_{l}^{(N) \star}, p_{l}^{(N)}, \alpha_{l}^{(N)}, \alpha_{l}^{(N) \star}\right\}$ of macroscopic variables is a Lie algebra w.r.t. commutation. We simplify our notation by defining $x^{(N)} \equiv\left(x_{1}^{(N)}, \ldots, x_{5 n}^{(N)}\right)$ to be the $5 n$ self-adjoint operators, which, by equns. (3.16)-(3.19), comprise the Hermitian and anti-Hermitian parts of the elements of $M^{(N)}$. It follows from this definition that the norms of the commutators of the these operators are all $O\left(N^{-1}\right)$, which suggests that their dynamics might become classical when $N \rightarrow \infty$. In anticipation of such a classical limit, we introduce a phase space, $X=\mathbf{R}^{5 n}$, and, for each point $x=\left(x_{1}, \ldots, x_{5 n}\right) \in X$, we define $\left\{\alpha_{l}, s_{l}, p_{l} \mid l=0, \ldots, n-1\right\}$ to be the complex numbers related to $x$ in precisely the same way that the elements of $M^{(N)}$ are to $x^{(N)}$. We define $\mathcal{C}_{0}(X)$ to be the space of bounded continuous functions on $X$, that tend to zero at infinity.

4. Dynamics, phase structure and a non-equilibrium entropy principle. We formulate the dynamics of the infinite system, $\Sigma$, as a limit of that of the sequence of finite systems, $\left\{\Sigma^{(N)} \mid N \in \mathbf{N}\right\}$, subject to the following conditions on their initial states, $\psi^{(N)}$.

(I.1) The mean photon number is not super-extensive, i.e.,

$$
\psi^{(N)}\left(a_{l}^{\star} a_{l}\right)<C N, \forall N \in \mathbf{N}, l \in[0, n-1],
$$

where $C$ is a finite constant.

(I.2) The mean and dispersion of each macroscopic observable $x_{j}^{(N)}$, for the state $\psi^{(N)}$, tend to limits $x_{j}$ and 0 , respectively, as $N \rightarrow \infty$. This macroscopic sharpness is characteristic of pure phases.

(I.3) There is a (unique) state, $\psi_{\text {mat }}$, on $\mathcal{A}_{\text {mat }}$, the algebra of observables of the infinite system $\Sigma_{\text {mat }}$, such that the restrictions $\psi_{\text {mat }}$ and $\psi^{(N)}$ to $\mathcal{A}_{\text {mat }}^{(N)}$ are identical, for each $N \in \mathbf{N}$. This condition is satisfied if, for example, $\psi^{(N)}=\psi_{\text {mat }}^{(N)} \otimes \psi_{\text {rad }}$, where $\psi_{\text {mat }}^{(N)}$ is the restriction of $\psi_{\text {mat }}$ to $\mathcal{A}_{\text {mat }}^{(N)}$.

4.1. The macroscopic dynamics. As in [6], we relate the macroscopic evolution of $\Sigma^{(N)}$, in the limit $N \rightarrow \infty$, to the following classical dynamical system.

Definition 4.1. We define $\mathcal{K}$ to be the flow in $X$, whose equation of motion,

$$
\frac{d x_{t}}{d t}=F\left(x_{t}\right)
$$

is of the explicit form

$$
\begin{gathered}
\frac{d \alpha_{l, t}}{d t}=-\left(i \omega_{l}+\kappa_{l}\right) \alpha_{l, t}+\lambda_{l} s_{l, t} \\
\frac{d s_{l, t}}{d t}=-\left(i \epsilon+\gamma_{1}\right) s_{l, t}+\sum_{m=0}^{n-1} \lambda_{m} p_{[l-m], t} \alpha_{m, t}
\end{gathered}
$$

and

$$
\frac{d p_{l, t}}{d t}=-\gamma_{2}\left(p_{l, t}-\eta \delta_{l, 0}\right)-2 \sum_{m=0}^{n-1} \lambda_{m}\left(\bar{\alpha}_{m, t} s_{[l+m], t}+\alpha_{m, t} \bar{s}_{[m-l], t}\right),
$$

where $[l \pm m]=l \pm m(\bmod n)$.

The following Propositions were proved in [6]. 
Proposition 4.2. The equation of motion (4.2) (i.e. (4.3)) has a unique global solution, corresponding to a one- parameter semigroup of transformations $x \rightarrow \tau(t) x\left(\equiv x_{t}\right)$ of $X$, that maps the compacts into compacts.

Proposition 4.3. Let $\mu_{t}^{(N)}$ be the time-dependent quantum characteristic function on $X$, defined by the formula

$$
\mu_{t}^{(N)}(y)=\psi_{t}^{(N)}\left(\exp \left(i y \cdot x^{(N)}\right)\right) \forall y \in X, t \in \mathbf{R}_{+} .
$$

Then, under the initial conditions $(I .1-3), \mu_{t}^{(N)}$ converges pointwise, as $N$ tends to infinity, to the characteristic function of the classical Dirac probability measure, $\delta_{x_{t}}$, on $X$, with support at $x_{t}=\tau(t) x$. Thus, the macroscopic dynamics reduces to that of the classical system $\mathcal{K}$.

Comment. This last Proposition signifies that the macroscopic observables $x^{(N)}$ reduce to the classical ones $x$, as $N$ tends to infinity. Correspondingly, the radiative field, $\phi^{(N)}$, reduces to the classical one, $\phi$, whose value at the site $r$ and time $t$ is

$$
\phi_{r, t}=-i \sum_{l=0}^{n-1} \lambda_{l} \alpha_{l, t} \exp (2 \pi i r l / n) .
$$

4.2. Phase structure. We see from Proposition 4.3 that, under the initial conditions (I.1-3), the time-dependent macroscopic properties of the system are governed by the classical dynamical system $\mathcal{K}$. A study of this system yields the following results [6].

(1) For $\eta$ less than a certain specified critical value, $\eta_{1}(>0)$, the system has a unique stable stationary state, corresponding to the fixed point, $x_{0}(\in X)$, given by

$$
\alpha_{l}=0 ; s_{l}=0 ; p_{l}=\eta \delta_{l 0} \forall l \in[0, n-1] .
$$

(2) As $\eta$ increases through a certain positive value, $\eta_{1}$, the fixed point $x_{0}$ becomes unstable, and, by Hopf bifurcation, gives way to a periodic orbit of the form

$$
\alpha_{l, t}=\alpha_{l}^{(0)} \exp \left(-i\left(\nu t+\theta^{(0)}\right)\right) \delta_{l k} ; s_{l, t}=s_{l}^{(0)} \exp \left(\left(-i \nu t+\theta^{(0)}\right)\right) \delta_{l k} ; p_{l, t}=\eta_{1} \delta_{l 0},
$$

where the selection of the $k$ 'th mode and the values of the constants $\alpha^{(0)}, s^{(0)}$ and $\nu$ are determined by the parameters of the model, while the phase angle $\theta^{(0)}$ is indeterminate. Thus, in optical terms, there is a transition from normal to coherent radiation as $\eta$ passes through $\eta_{1}$. This entails a breakdown of the gauge symmetry, represented by the transformations in which the $\sigma_{r,-}, a_{l}$, and correspondingly $s_{l}, \alpha_{l}$, are all rephased by the same factor $\exp (i \theta)$.

(3) The model generically undergoes a further transition from coherent to chaotic radiation, as $\eta$ passes through a second critical value, $\eta_{2}\left(>\eta_{1}\right)$. Specifically, the following two scenarios, both of which involve gauge symmetry breakdown, are feasible.

(a) There can be a bifurcation, at $\eta=\eta_{2}$, from a periodic orbit to a strange attractor, as specified in the Ruelle-Takens scheme [15]. This scenario actually realised in the single mode case [6].

(b) For large $n$, there could be a succession of bifurcations, corresponding to the activation of different modes, leading to a chaotic phase along the lines of Landau's theory of hydrodynamical turbulence [17]. 
Comment. These results signify that the model satisfies the conditions (I) and (II) of the scheme of Section 2.3, with $\eta$ playing the role of the control variable $\theta$. The control space, $\Theta$, is simply the interval, $[-1,1]$, over which the value of this variable may range.

4.3. The microscopic dynamics. We take the local microscopic observables to be those of the matter only, since, as noted in the Comment following Proposition 4.3, the radiation field of the model reduces to a classical macroscopic one in the limit $N \rightarrow \infty$.

The following two Propositions were proved in [7]. The first one signifies that, in the limit $N \rightarrow \infty$, the microscopic dynamics is simply that of $\Sigma_{m a t}$, under the action of the classical macroscopic radiation field, $\phi$. The second one substantiates that the model satisfies the conditions (III), (IV) of Section 2.3.

Proposition 4.4. (i) Under the conditions (I.1-3), the microscopic dynamics of the model corresponds, in the limit $N \rightarrow \infty$, to a two-parameter family,

$$
\left\{\mathcal{T}(s, t \mid \phi) \mid 0 \leq s \leq t ; \mathcal{T}(s, u \mid \phi) \mathcal{T}_{\mu}(u, t \mid \phi)=\mathcal{T}(s, t \mid \phi)\right\},
$$

of $C P$ contractions of $\mathcal{A}_{\text {mat }}$, in that

$$
\lim _{N \rightarrow \infty} \psi^{(N)}\left(T^{(N)}(t) A\right)=\psi_{\text {mat }}(\mathcal{T}(0, t \mid \phi) A) \forall A \in \mathcal{A}_{L}, t \in \mathbf{R}_{+} .
$$

Furthermore, the generator of $\mathcal{T}$ is

Thus,

$$
\mathcal{L}(t \mid \phi):=\frac{\partial}{\partial t} \mathcal{T}(s, t \mid \phi)_{\mid s=t}=L_{m a t}+\sum_{r \in \mathbf{Z}}\left[\phi_{r, t} \sigma_{r,+}-\text { h.c. }, .\right] .
$$

$$
\mathcal{T}(s, t \mid \phi)=\mathbf{T}_{a} \exp \left(\int_{s}^{t} d u \mathcal{L}(u \mid \phi)\right)
$$

where $\mathbf{T}_{a}$ is the antichronological operator. We define

$$
\psi_{t, \text { mat }}:=\psi_{\text {mat }} \circ \mathcal{T}(0, t) .
$$

Proposition 4.5. (i) Following the exponential decay of transients in the microscopic relaxation time $\max \left(\gamma_{1}^{-1}, \gamma_{2}^{-1}\right)$, $\psi_{\text {mat, } t}$ reduces to a state, $\omega_{s_{t}, p_{t}}$, which is completely determined by the instantaneous values of the macroscopic variables $s_{t}$ and $p_{t}$.

(ii) This state, like the classical field, $\phi$, is spatially periodic, with periodicity $n$.

(iii) Further, it maximises the entropy density of $\Sigma_{\text {mat }}$, as defined on the states with this spatial periodicity, subject to the constraints imposed by the prevailing instantaneous values of the macroscopic variables $s_{t}$ and $p_{t}$.

5. Discussion of results. We see from Propositions 4.3 and 4.5 , together with the results described in Section 4.2, that the laser model conforms to the scheme proposed in Section 2.3 for non-equilibrium phase structures in dissipative systems. It is not difficult to construct other mean field theoretic models which would yield the same results. For example, a class of such models is given by equipping $\Sigma_{m a t}^{(N)}$ with a self-interaction energy of the form $N F\left(s^{(N)}, s^{(N) \star}, p^{(N)}\right)$, with $s^{(N)}$ and $p^{(N)}$ defined as previously and the function $F$ carrying no explicit dependence on $N$.

Although all these models are rather primitive, one might envisage that counterparts of our results might have at least some measure of validity in the physics of real dissipative 
systems. For example, one might expect a local version of the maximum entropy principle, given by Proposition 4.5 (iii) to prevail in hydrodynamics, in such a way as to impose local equilibrium conditions on the states of fluids.

\section{References}

[1] R. Graham and H. Haken, Z. Phys. 237 (1970), 31.

[2] H. Haken, Handbuch der Physik, Bd. XXV/2C, Springer, Heidelberg, Berlin, New York, 1970; and Rev. Mod. Phys. 47 (1975), 67.

[3] P. Glansdorff and I. Prigogine, Thermodynamic Theory of Structure, Stability and Fluctuations, Wiley-Interscience, London, New York, Sidney, Toronto, 1974.

[4] H. Fröhlich, Int. J. Quant. Chem. 2 (1968), 641.

[5] K. Hepp and E. H. Lieb, Helv. Phys. Acta 46 (1973), 573; and J. Moser (ed.), Dynamical Systems, Theory and Applications, Springer Lecture Notes in Physics 38, Springer, Heidelberg, New York, 1975, pp. 178-208.

[6] G. Alli and G. L. Sewell, J. Math. Phys. 36 (1995), 5598.

[7] F. Bagarello and G. L. Sewell, J. Math. Phys. 39 (1998), 2730.

[8] G. L. Sewell, Helv. Phys. Acta 67 (1994), 4; and Lett. Mat. Phys. 40 (1997), 203.

[9] N. G. Duffield, Phys. Lett. 110 A (1985), 332; and J. Phys. A 21 (1988), 625.

[10] D. Ruelle, Statistical Mechanics, W. A. Benjamin Inc., New York, 1969.

[11] G. G. Emch, Algebraic Methods in Statistical Mechanics and Quantum Field Theory, J. Wiley, New York, London, 1972.

[12] G. L. Sewell, Quantum Theory of Collective Phenomena, Oxford University Press, Oxford, New York, Toronto, 1989.

[13] O. E. Lanford and D. Ruelle, Commun. Math. Phys. 13 (1969), 194.

[14] H. Callen, Thermodynamics and an Introduction to Thermostatistics, J. Wiley, New York, Chichester, Brisbane, 1985.

[15] D. Ruelle and F. Takens, Commun. Math. Phys. 20 (1971), 167.

[16] P. Vanheuverzwijn, Ann. Inst. H. Poincare A 29 (1978), 123; Erratum ibid 30 (1979), 83; and B. de Moen, P. Vanheuverzwijn and A. Verbeure, Rep. Math. Phys. 15 (1979) 27.

[17] L. D. Landau and E. M. Lifshitz, Fluid Mechanics, Pergamon, Oxford, New York, Toronto, Sydney, Paris, 1984. 